\title{
Un modèle intégré pour explorer les trajectoires d'utilisation de l'espace
}

\author{
Eric Fotsing ${ }^{1,5, *}$, Peter H. Verburg ${ }^{2}$, Wouter T. De Groot ${ }^{3}$, Jean-Paul \\ Cheylan $^{4}$, Maurice Tchuente ${ }^{5}$ \\ ${ }^{1}$ Département d'Informatique, IUT/Fotso Victor de Bandjoun, Université de Dschang, Cameroun \\ ${ }^{2}$ Institute for Environmental Studies, VU University Amsterdam, Amsterdam, the Netherlands, \\ ${ }^{3}$ Institute of Environmental Science, University of Leiden, The Netherlands \\ ${ }^{4}$ CIRAD-TERA/CNRS - UMR ESPACE, Montpellier, France \\ ${ }^{5}$ LIRIMA, Equipe IDASCO, Département d'Informatique, Faculté des sciences, Université de \\ Yaoundé I, IRD UMI 209 UMMISCO, Yaoundé - Cameroun \\ *Corresponding author : efotsing@gmail.com
}

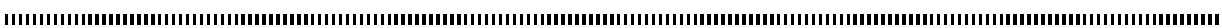
RÉSUMÉ. Les modèles spatiaux dynamiques sont des outils de très grande importance pour l'étude des systèmes complexes comme les systèmes environnementaux. Cet article présente un modèle intégré développé pour explorer les trajectoires d'utilisation de l'espace dans une petite région autour de la ville de Maroua, dans l'Extrême Nord du Cameroun. Le modèle simule la compétition entre différentes catégories d'utilisation de l'espace en prenant en compte l'influence d'un ensemble de facteurs biophysiques, socio-démographiques et géoéconomiques. On distingue trois principaux modules. Le module de simulation dynamique combine les résultats des modules d'analyse spatiale et de prédiction. Les résultats de simulation pour chaque scénario permettent d'identifier des zones où s'opèrent les changements. Le modèle développé constitue ainsi un outil de recherche exploratoire et un support de connaissances utilisable pour la planification de l'utilisation de l'espace.

ABSTRACT. Dynamic spatial models are important tools for the study of complex systems like environmental systems. This paper presents an integrated model that has been designed to explore land use trajectories in a small region around Maroua, located in the far north of Cameroon. The model simulates competition between land use types taking into account a set of biophysical, socio-demographic and geo-economics driving factors. The model includes three modules. The dynamic simulation module combines results of the spatial analysis and prediction modules. Simulation results for each scenario can help to identify where changes occur. The model developed constitutes an efficient knowledge support system for exploratory research and land use planning.

MOTS-CLÉS : changements d'utilisation de l'espace, modèle intégré, analyse spatiale, trajectoire, scénario, transition agraire.

KEYWORDS: land use change, integrated model, spatial analysis, trajectory, scenario, agrarian transition

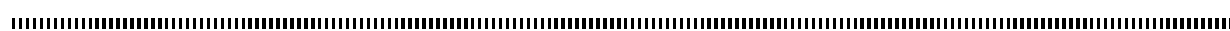




\section{Introduction}

La région de l'Extrême Nord du Cameroun est un exemple de région de savanes sèches d'Afrique centrale qui est confrontée au défi de l'explosion démographique qui rend les changements inéluctables. On a observé au cours des deux dernières décennies d'importantes transformations du paysage agraire et de l'utilisation de l'espace [5]. La réduction des espaces de brousse conduit à une compétition pour l'espace entre les activités agricoles, pastorales et sylvicoles et nécessite d'adapter les politiques de gestion de l'espace et des ressources naturelles. En particulier, les superficies nécessaires à la continuation du système extensif actuel sont devenues très limitées en mais les prévisions fournies par le schéma d'aménagement de la région et le dernier recensement envisagent un doublement de la population dans les trois prochaines décennies [20]. Une question importante pour le développement de cette région est de connaître les stratégies que les populations pourront mettre en place pour satisfaire leurs différents besoins en aliment et en bois de feu dans un futur proche. La réponse à cette question nécessite d'identifier les principaux facteurs et les mécanismes qui déterminent les changements d'utilisation de l'espace avant d'envisager toute analyse prospective [6].

Les modèles de simulation sont des outils appropriés pour l'étude des systèmes complexes. Les modèles dynamiques peuvent permettre de saisir la complexité qui émerge des systèmes d'utilisation de l'espace et analyser les trajectoires d'évolution possibles en offrant la possibilité de tester la sensibilité de la structure et la stabilité de l'ensemble du système face aux changements des facteurs déterminants [23]. Ces modèles fournissent ainsi des informations qui peuvent permettre d'évaluer des options d'aménagement et de gestion de l'espace, d'apprécier les impacts des changements d'utilisation de l'espace sur l'environnement naturel, sur les conditions socio-culturelle, économique ou politique et inversement. Une très large gamme de modèles appliqués à l'utilisation de l'espace a été développée pour répondre à une diversité de besoins ([1] ; [9]; [10]; [15]). Une approche de modélisation intégrée est indispensable lorsqu'on veut avoir une compréhension plus complète du comportement des systèmes complexes [21]. Dans cet article, nous décrivons les bases du modèle dynamique développé pour explorer les trajectoires des changements d'utilisation de l'espace dans la région autour de Maroua, à l'Extrême Nord du Cameroun [4].

La section suivante présente la zone d'étude et les principales dynamiques d'utilisation de l'espace. La section 3 est consacrée à la formulation des principales hypothèses. La section 4 justifie l'approche de modélisation et la section 5 décrit la structure du modèle de simulation développé. Cette section présente tour à tour les différents modules, la calibration et la validation du modèle. La section 6 analyse les 
résultats de simulation des différents scénarios formulés. La section 7 rappelle les leçons apprises et dégage quelques perspectives d'amélioration et de valorisation du modèle.

\section{Localisation de la zone d'étude et dynamiques du système d'utilisation de l'espace}

La zone d'étude pour cette application est une région autour de la ville de Maroua, le principal centre urbain de la région de l'Extrême Nord du Cameroun. L'étendue de cette zone qui correspond à celle d'une scène d'image du satellite SPOT, couvre une superficie d'environ $3600 \mathrm{~km}^{2}$ (figure 1). Cette région peut être considérée comme une unité géographique représentative de la zone des savanes de l'Extrême Nord du Cameroun du fait qu'elle renferme la diversité des situations agroécologiques identifiées à l'échelle régionale. Ainsi, elle est partagée entre la plaine du Diamaré autour de Maroua, la pédiplaine de Kaélé et les piémonts des monts Mandara autour de Mowo. C'est une zone de forte densité de population avec d'importants flux migratoires. La répartition de la population n'est pas uniforme. La densité moyenne estimée à 50 hab. $/ \mathrm{km}^{2}$ en 1987 est passée à 75 hab. $/ \mathrm{km}^{2}$ en 2002 et on atteint $300 \mathrm{hab} . / \mathrm{km}^{2}$ par endroits. La population essentiellement rurale a été estimée à $80 \%$ de la population totale [20].

Le système agraire est caractérisé par une importante activité pastorale et un secteur agricole en pleine expansion. Le cotonnier est resté longtemps la principale culture de rente. Mais sa production est toutefois marginale au regard des rendements et de la production de l'ensemble des zones de savanes d'Afrique Centrale. La production vivrière est assez forte, associée à l'élevage qui est considéré comme une source d'investissement importante. On note qu'une très grande diversité de cultures est pratiquée parmi lesquelles le sorgho pluvial, le sorgho de contre saison, le maïs, l'arachide, le haricot, le riz, et l'oignon. Le schéma d'aménagement de la région a montré en 1992 qu'environ 15\% des sols apparait dénudés et correspond dans la plupart des cas à des sols dégradés qui ne sont plus propices à l'agriculture. La tendance à la dégradation s'est renforcée avec la pression démographique et les pratiques agricoles extensives non respectueuses de l'environnement qui entrainent la baisse de la fertilité des sols [17]. L'un des problèmes assez préoccupant de cette région est relatif aux faibles rendements de la production agricole qui est restée presque stable au cours des trois dernières décennies [14] alors que les besoins de la population ont continué à augmenter du fait de la croissance démographique. Les populations en quête d'espace cultivable et de pâturage exercent des pressions importantes sur les terres qui conduisent à la saturation foncière [22], à la dégradation des sols et des ressources ligneuses [11]. A l'origine de ces transformations, on peut également citer à côté de la très forte pression démographique, les courants migratoires qui ont marqué l'histoire de cette région, le 
déplacement des troupeaux venant des zones sahéliennes à la recherche de nouveaux pâturages, le problème que pose l'accès au bois de feu par les ménages et son impact sur les zones d'approvisionnement. Le foisonnement de tous ces processus au sein d'un même territoire est de nature à déboucher sur des conflits d'activités, la dégradation de la productivité du milieu et la perte de sa biodiversité.

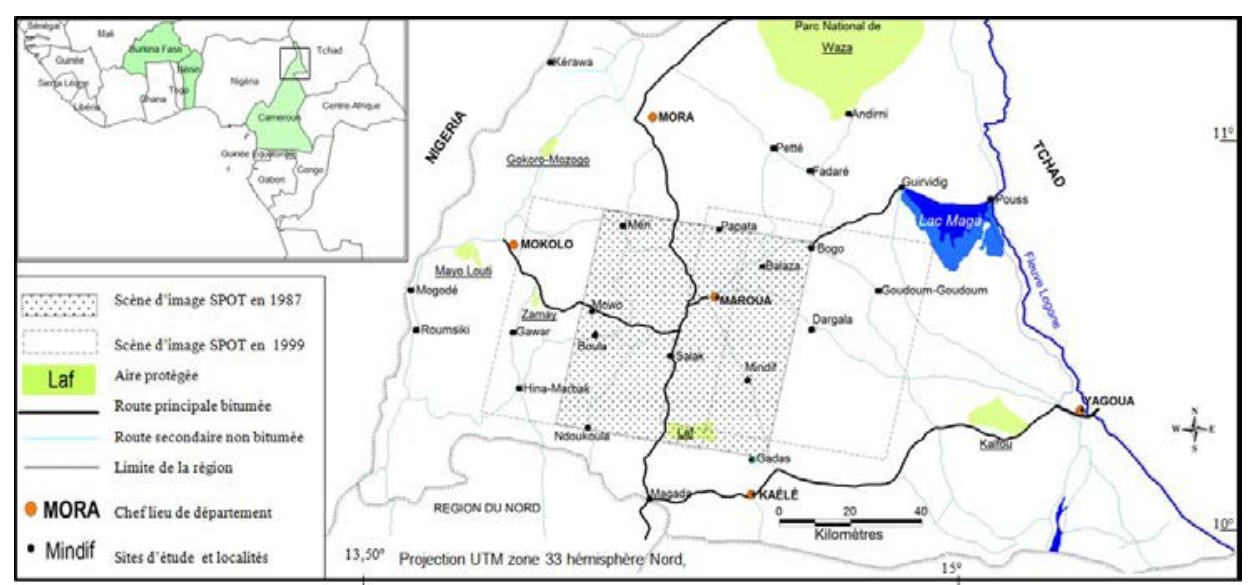

Figure 1. Localisation de la zone d'étude et des scènes d'image satellite analysées

\section{Hypothèses sur l'évolution des changements d'utilisation de l'espace}

Dans la plupart des zones de savane de l'Extrême Nord du Cameroun, les processus de pression démographique et de saturation foncière se sont opérés de manière similaire aux situations survenues un peu plus tôt dans de nombreuses régions en Afrique de l'Ouest ou soudano-sahélienne [17]. En effet, la réponse la plus couramment observée à l'accroissement de la population dans ces régions a pendant longtemps été l'extension des surfaces cultivées. Le modèle descriptif des dynamiques en cours présenté dans [8] met en exergue trois principaux processus: l'extension des terres cultivables, la disparition des formations boisées de savanes, la dégradation et la raréfaction des zones de pâturages. La figure 2 illustre les principales phases du processus de saturation foncière représentées par des portions de la courbe d'évolution de l'espace libre et utile en fonction de la population. Le terme utile précise le fait que la saturation ne s'exprime pas seulement en terme de disponibilité quantitative mais également et surtout qualitative. En effet, les espaces de savanes non cultivés jouant le rôle de réserve 
foncière ont été progressivement exploités pour répondre à l'accroissement des besoins et pour absorber une force de travail en augmentation [16]. Avec la croissance continue et rapide de la population, l'intensité culturale mesurée comme le rapport entre le nombre d'années de mise en culture et la durée totale, a tendance à s'accroître et on passe ainsi progressivement d'une agriculture itinérante à un système agraire spatialement saturé [13]. On est parvenu dans la plupart des terroirs à un niveau de saturation de l'espace «utile» qui se traduit par la mise en valeur des terres jugées marginales.

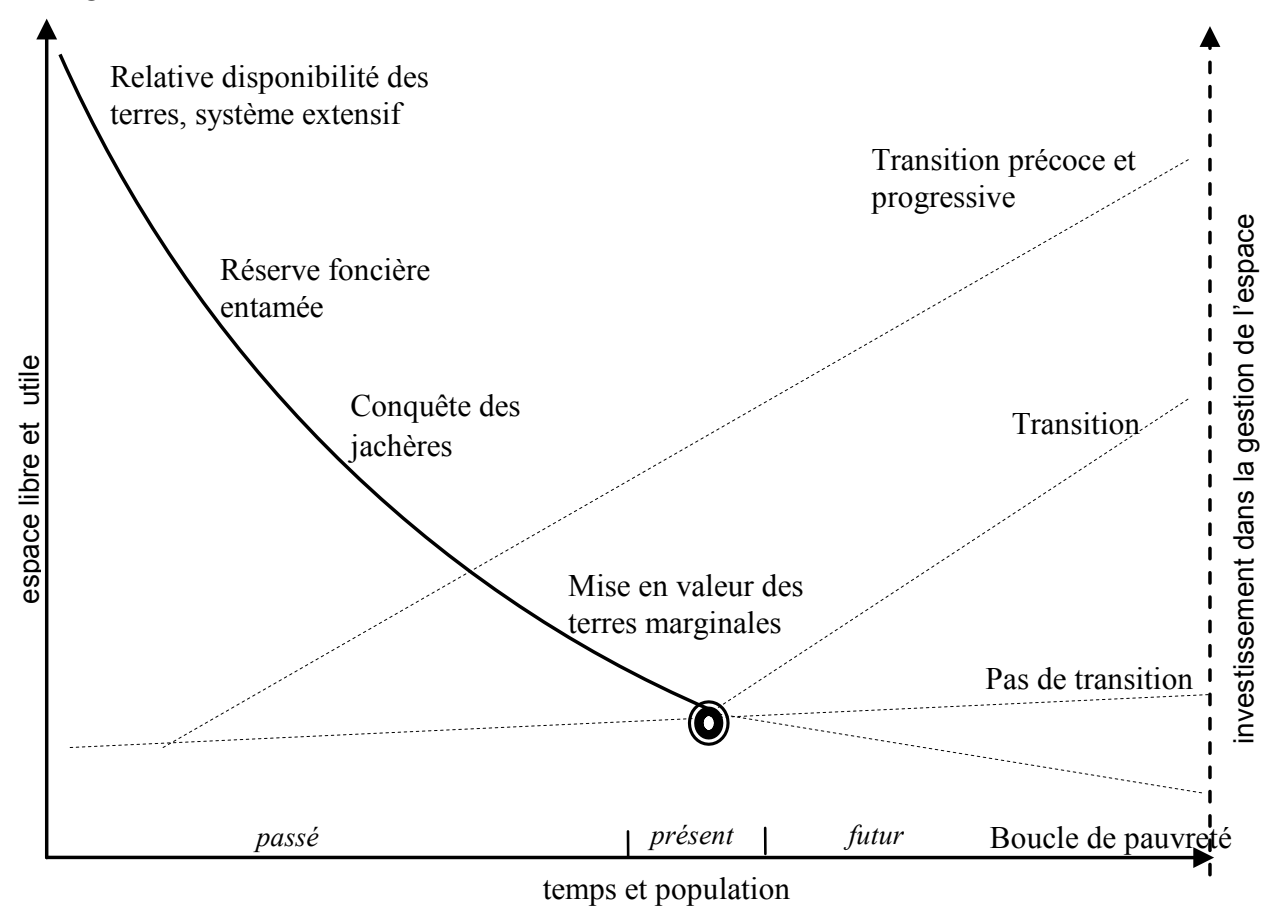

Figure 2. Les processus de changement d'utilisation de l'espace et trajectoires hypothétiques dans un futur proche

Les superficies nécessaires à la continuation du système extensif observé sont devenues très limitées mais les prévisions indiquent que la population de cette région devra encore doubler dans les trois prochaines décennies [20]. Une question importante pour les acteurs impliqués dans le développement de la région est de savoir quelles sont les dynamiques d'utilisation de l'espace qui vont se mettre en place et comment les populations devront faire face à ces mutations pour satisfaire leurs besoins alimentaires. Les principales hypothèses formulées sur la trajectoire des changements d'utilisation de 
l'espace dans le futur font référence aux théories de Boserup et Malthus ([2], [12]; [13]). La visoin néo-Malthusienne est une vision pessimiste, le plus souvent soutenue par les biologistes ou écologistes qui soutiennent que les ressources naturelles sont finies et déterminent des limites strictes de croissance démographique et de consommation globale [12]. L'augmentation de la population et les pratiques extensives d'utilisation des terres actuelles devraient donc conduire à une dégradation continue du milieu productif et pourrait à son tour renforcer la pauvreté, provoquer les famines et l'exode.

Par contre, la thèse de Boserup [2] correspond à une vision optimiste, le plus souvent soutenue par les économistes qui pensent que très peu sinon presque aucune société ne présente des limites strictes de population ou de consommation. Par conséquence, les crises de subsistance pourraient multiplier les tentatives innovatrices qui peuvent déboucher sur une intensification durable des systèmes de production.

Les dynamiques d'utilisation durable de l'espace dans un futur proche vont dépendre principalement des réponses paysannes en termes d'investissement dans la conservation et la gestion des ressources naturelles. Ces investissements concernent notamment l'amélioration de la qualité des sols et des institutions qui gouvernent l'utilisation de l'espace comme le régime foncier, le marché ou les pratiques paysannes, etc. L'analyse spatiale effectuée sur les images de la zone d'étude dans [8] montrent que les extensions récentes de la culture du sorgho de contre saison se font sur des terres marginales qui nécessitent des investissements importants. Njomaha [14] a étudié une vingtaine de village de la région de l'Extrême Nord du Cameroun pour lesquels il évalue les contraintes et les atouts de chacun pour de tels investissements en s'appuyant sur les résultats d'enquêtes auprès des producteurs. Il conclut que la région dans l'ensemble semble s'orienter vers une trajectoire Malthusienne. Toutefois, ses résultats mettent en exergue quelques sites comme Meskine dans la plaine du Diamaré qui présentent des signes d'une trajectoire Boserupienne. Dans ce contexte, la modélisation dynamique des changements d'utilisation de l'espace à l'échelle régionale est très importante. Elle devrait permettre d'explorer les conditions d'évolution de chaque site et de proposer des solutions pour éviter que le système ne s'oriente vers une trajectoire indésirable.

\section{Justification de l'approche de modélisation}

Dans la littérature sur les approches d'analyse et de modélisation on retrouve plusieurs typologies des modèles en fonction des objectifs. La typologie que nous retenons dans cette étude compte principalement trois classes de modèles qui se distinguent par la manière dont les processus et les mécanismes de changement sont appréhendés : les modèles empiriques, les modèles mécanistes (déterministes) et les modèles de type système [9]. Ces modèles utilisent une grande variété de techniques de 
prédiction et de simulation des processus de changement telles que les régressions statistiques, les probabilités de transition, les équations différentielles, la programmation linéaire, et les systèmes multi-agents. Au regard de cette typologie on peut anticiper que le modèle le plus utile pour mieux analyser les dynamiques d'utilisation de l'espace et explorer les scénarios à venir devrait reposer sur une approche hybride ou intégrée ([21]; [10]). Une telle approche tire profit plus ou moins de plusieurs approches et se trouve indispensable lorsqu'on veut avoir une compréhension plus complète du comportement d'un système complexe. Le but ultime de ce travail est de construire et mettre en œuvre un tel modèle dynamique pour la région d'étude. Notre démarche part d'une approche et explore ensuite les possibilités de complémentarité avec les autres approches. Les approches empiriques qui sont généralement de type statistique apparaissent comme un bon point de départ dans le contexte de la zone d'étude qui est assez complexe et où on ne dispose pas assez de connaissances formelles sur le fonctionnement du système d'utilisation de l'espace pour envisager une approche déterministe ou systémique.

L'approche de modélisation suivie est donc principalement de type spatial et empirique. Elle combine à cet effet les données de la télédétection sous un SIG avec des modèles mathématiques multi-variés ou multi-temporels. Les changements d'utilisation de l'espace sont modélisés comme la fonction d'une sélection de variables socioéconomiques et biophysiques qui agissent comme des facteurs déterminant les changements d'utilisation. Une analyse des changements observés dans la structure de l'utilisation de l'espace basée sur les techniques statistiques permet de sélectionner les facteurs déterminants les plus importants et de quantifier les relations. La mise en œuvre dans les modèles dynamiques varie d'une translation directe de l'analyse empirique en probabilités de transition [1] aux modèles intégrés combinant les relations déterminées empiriquement avec une modélisation mécaniste des interactions entre les types d'utilisation de l'espace et les rétroactions dans le système [24]. La principale hypothèse de ce type de modèles basés sur une analyse empirique des facteurs déterminants c'est que la structure des facteurs déterminants est la même au cours du temps de simulation. C'est pourquoi les prédictions avec ces modèles sont le plus souvent limitées aux changements dans un futur proche (entre 10 et 20 ans). Toutefois, l'intérêt de ce type de modèle réside dans sa capacité à simuler les structures d'utilisation de l'espace et localiser les changements qui résulteraient de la continuation des pratiques actuelles de gestion de l'espace. 


\section{Structure et mise en œuvre du modèle de simulation}

\subsection{Aperçu général de la structure du modèle}

Le modèle développé est composé de trois principaux modules tels que présentés sur la figure 3 et explicités sur les étapes du processus de modélisation illustrées par la figure 4.

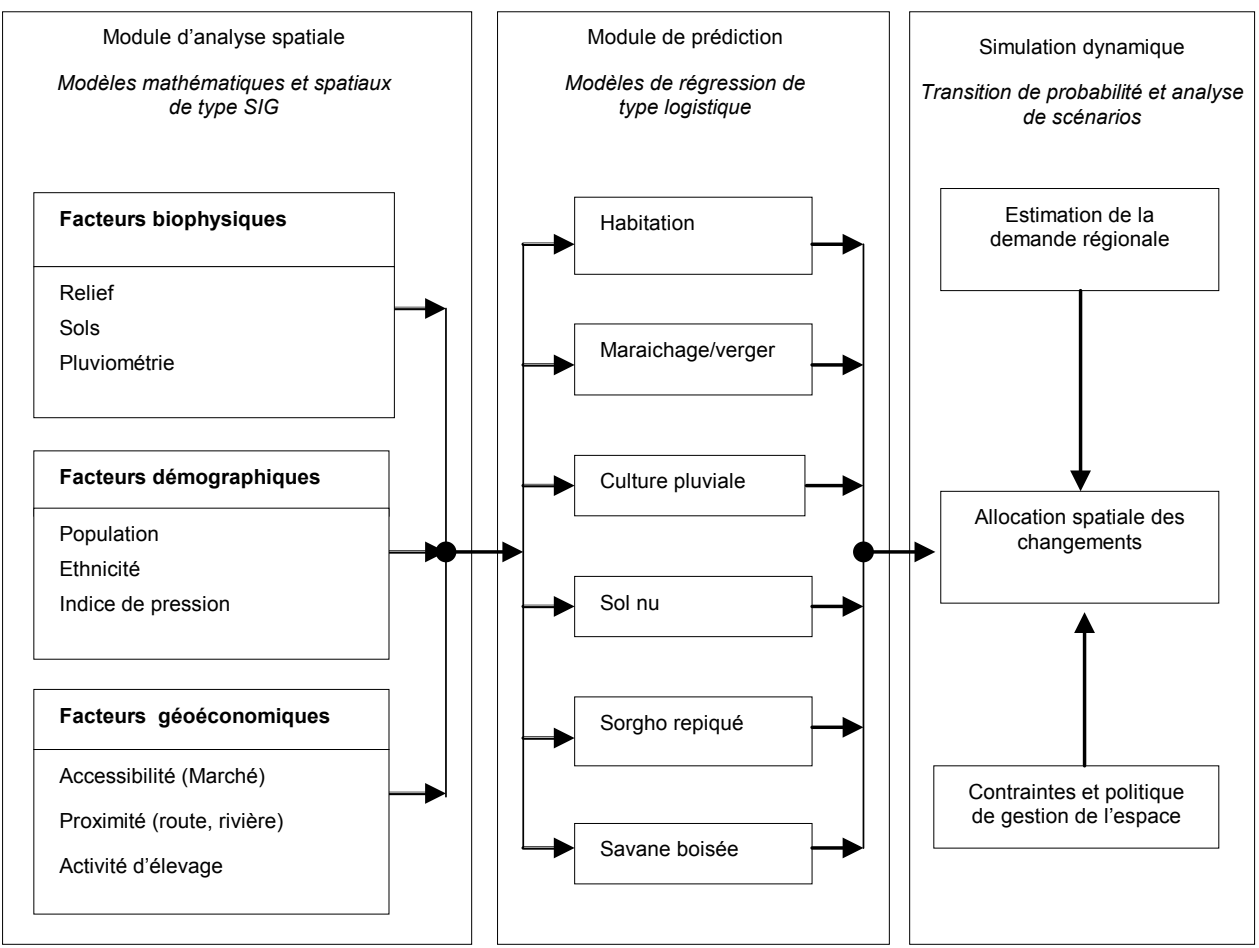

Figure 3. Structure du modèle intégré de changement d'utilisation de l'espace sur la région autour de Maroua.

Le module d'analyse spatiale repose sur les logiciels ILWIS pour l'analyse d'images et ArcGIS pour les analyses spatiales. Il est constitué de sous-modèles mathématiques qui définissent les variables représentant la distribution de l'utilisation de l'espace et des facteurs déterminants. Les données étant de sources variées (recensement de la population, MNT, relevés pluviométriques, cartes existantes des sols et des cours d'eau, 
etc.), des traitements appropriés ont été effectués sur chaque thème afin de construire une base de données géographiques. [6] décrit en détail les sources de données, les traitements effectués et les procédures de calcul des variables spatiales explicites. Les images satellites utilisées sont des scènes du capteur SPOT prises en saison sèche $(15 / 01 / 1987-28 / 02 / 1999)$ avec une résolution de $20 \mathrm{~m}$. La méthode de classification, publiée dans [5] combine itérativement traitement d'image, vérité de terrain, cartographie participative et photo-interprétation pour extraire des unités d'utilisation de l'espace pertinentes. Toutes les couches de données biophysiques, sociodémographiques et géoéconomiques sont ensuite ramenées au même système de référence et à la même résolution $(250 \mathrm{~m})$.

Le module de prédiction repose sur le logiciel SPSS pour l'analyse statistique. Il est constitué des modèles de prédiction de type régression logistique, définis pour chaque utilisation de l'espace. La procédure de construction des modèles de prédiction et les résultats obtenus sont décrits en détail dans [6]. Les coefficients logistiques obtenus sont utilisés pour calculer la probabilité d'allocation de chaque type d'utilisation de l'espace.

Le module de simulation dynamique implémenté sous la plateforme CLUE (Conversion of Land Use and its Effects), repose sur une procédure d'allocation spatiale des changements qui prend en entrée la demande régionale, les probabilités d'allocation et les paramètres de conversion de chaque utilisation de l'espace [24]. Pour chaque pas de temps, la demande de chaque utilisation de l'espace à l'échelle de la région est calculée au préalable par un modèle qui dépend du scénario exécuté. En effet, la demande qui correspond aux besoins futurs des populations en produits est traduite en superficies pour chaque scénario en fonction des hypothèses retenues. Une nouvelle carte de probabilité d'allocation de chaque utilisation de l'espace est également calculée en utilisant les valeurs mises à jour des facteurs déterminants qui changent dans le temps tels que la distribution de la population ou l'accessibilité (Figure 5).

La décision de conversion d'une utilisation de l'espace par le modèle prend aussi en compte les règles de conversion propre à chaque forme d'utilisation de l'espace comme l'élasticité et les séquences de conversion ou transition. Ces règles sont des caractéristiques intrinsèques du système d'utilisation de l'espace qui traduisent les mécanismes de changement. L'élasticité est un facteur de résistance au changement qui est affecté à chaque utilisation de l'espace en s'appuyant sur les connaissances du système d'utilisation de l'espace de la région ([5]; [8]). Elle correspond à la réversibilité des changements et traduit la facilité d'une cellule à passer d'un type à un autre. 


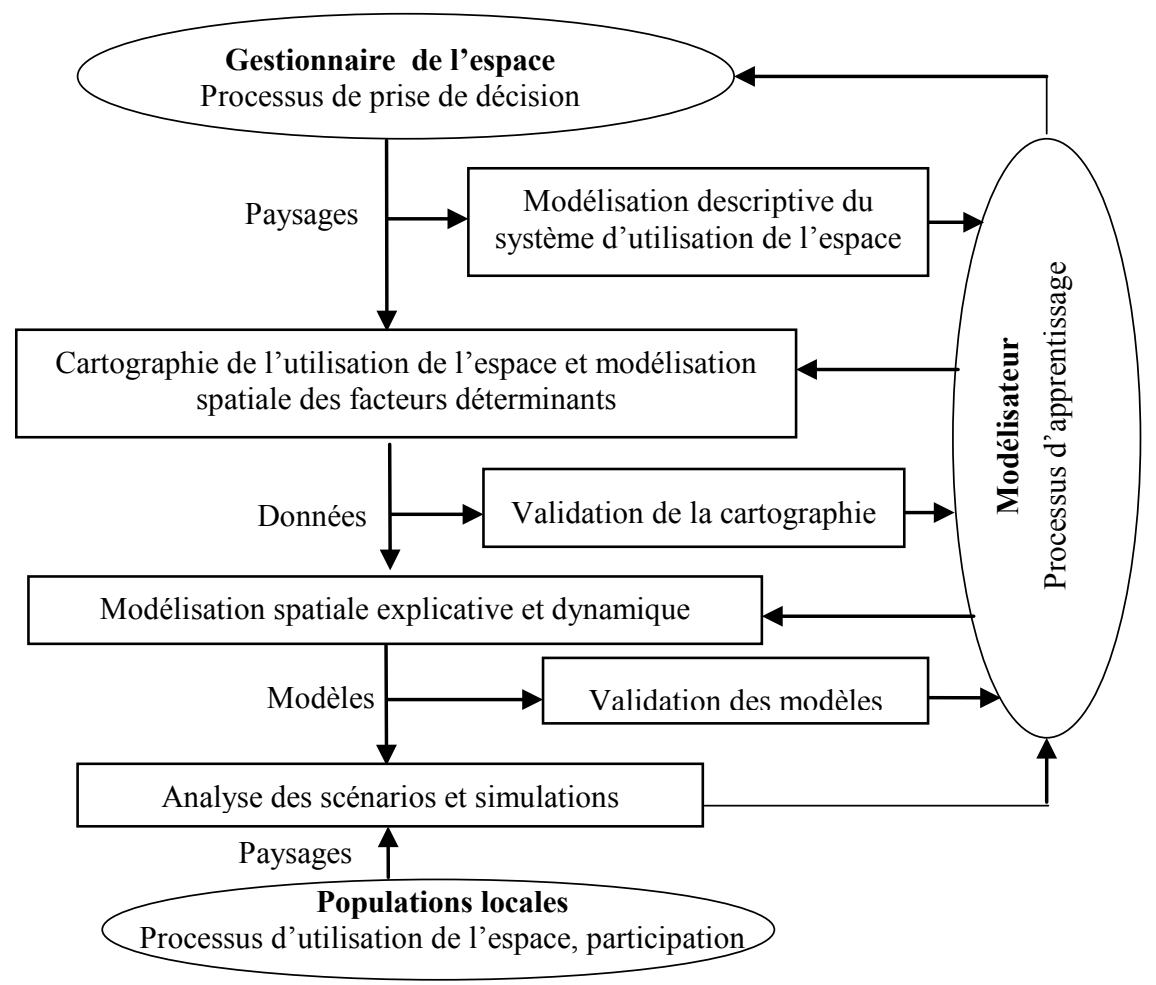

Figure 4. Synthèse des étapes du processus de modélisation. Les utilisateurs des modèles sont représentés en ovales et les étape de modélisation en rectangle.

Les séquences de conversion ou transition sont représentées par une matrice de transition où les valeurs indiquent les possibilités de conversion de chaque utilisation de l'espace vers une autre pour chaque pas de temps (tableau 1). Dans cette première version du modèle, on suppose que presque tous les changements sont possibles à chaque pas de temps, à l'exception de la classe montagne qui reste statique. D'autre part les abandons d'habitation observés localement sur les images satellites et confirmés à l'échelle des terroirs ont été négligés du fait qu'elles sont de très faible importance et difficilement interprétables à l'échelle de la région. La durée maximale ou minimale avant qu'une conversion ne prenne place n'est pas prise en compte dans cette version du modèle. Des restrictions spécifiques ou politiques de gestion de l'espace peuvent également être formulées pour chaque scénario. Par exemple, on peut définir une mesure 
de conservation consistant à interdire des défrichements dans certaines zones comme les aires protégées ou des zones de brousse. Il suffit dans ce cas de fournir une carte de ces espaces en entrée de la procédure d'allocation.
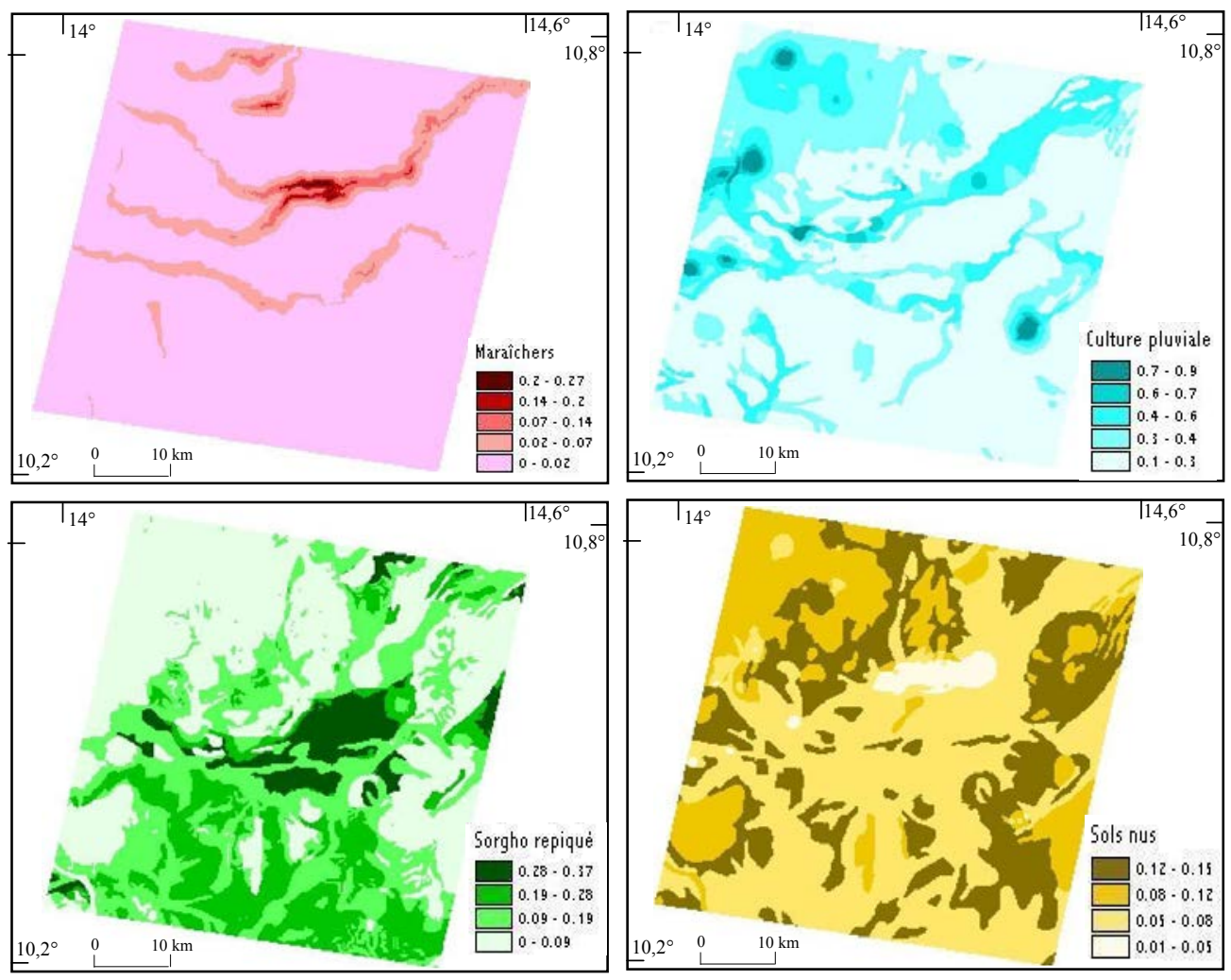

Figure 5. Cartes de probabilité d'allocation de quelques utilisations de l'espace (les zones qui ont une forte probabilité d'être allouées à chaque utilisation de l'espace en couleur foncé).

\subsection{Calibration du modèle}

La calibration est une procédure itérative où plusieurs simulations des changements entre 1987 et 1999 sont effectuées en modifiant les valeurs de l'élasticité jusqu'à obtenir une certaine stabilité de la structure du système d'utilisation de l'espace. Les paramètres de régression pour la situation de 1987 sont utilisés pour la calibration et ceux de 1999 
sont utilisés ultérieurement pour la prédiction [6]. Le tableau 2 donne les valeurs d'élasticité finalement retenues. Ces valeurs sont comprises entre 0 et 1.

Tableau 1. La matrice de transition du modèle de changement de l'utilisation de l'espace. ( 0 = changement impossible et $1=$ changement possible) N.B : Le pas de temps est annuel

\begin{tabular}{|c|c|c|c|c|c|c|c|c|}
\hline & \multicolumn{7}{|c|}{ Utilisation de l'espace à $\mathrm{t}+1$} \\
\hline & & 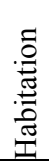 & 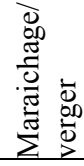 & 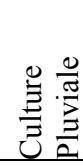 & $\begin{array}{l}\vec{\Xi} \\
\vec{\rho} \\
\dot{n}\end{array}$ & 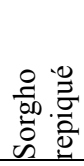 & 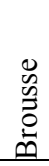 & 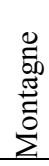 \\
\hline & Habitation & 1 & 0 & 0 & 0 & 0 & 0 & 0 \\
\hline & Maraichage/verger & 1 & 1 & 1 & 1 & 1 & 1 & 0 \\
\hline$\ddot{8}$ & Culture Pluviale & 1 & 1 & 1 & 1 & 1 & 1 & 0 \\
\hline 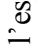 & Sol nu & 1 & 1 & 1 & 1 & 1 & 1 & 0 \\
\hline ฮี & Sorgho repiqué & 1 & 1 & 1 & 1 & 1 & 1 & 0 \\
\hline$\stackrel{\mathscr{\varrho}}{\Xi}$ & Brousse & 1 & 1 & 1 & 1 & 1 & 1 & 0 \\
\hline & Montagne & 0 & 0 & 0 & 0 & 0 & 0 & 1 \\
\hline
\end{tabular}

Les valeurs proches de 1 correspondent aux utilisations de l'espace qui changent difficilement. Par exemple, les zones de montagne principalement rocheuses ont une élasticité de 1 puisqu'elles sont statiques au cours de la simulation. Les zones d'habitation ont également une élasticité de 1 puisque les pixels qui se convertissent en zone d'habitation à une date donnée sont supposés garder cet état aux dates ultérieures. Par contre les zones de culture pluviale et les sols nus ont une élasticité de 0,2 parce qu'elles sont les classes qui changent les plus facilement. En effet, un pixel qui passe par exemple de l'état «brousse » à l'état « culture pluviale » peut, après cette conversion, être abandonné ou mise en jachère ( $s o l \mathrm{nu}$ ), utilisé pour l'extension de l'habitat ou pour l'agriculture de contre saison en fonction des besoins et des conditions du milieu.

Les zones de culture du sorgho repiqué ont une élasticité un peu plus grande parce qu'un pixel qui est occupé par le sorgho repiqué aura un peu plus difficilement tendance à se convertir à cause des contraintes d'exploitation des espaces réservés à cette culture pour d'autres formes de mise en valeur (agriculture pluviale ou habitation). De plus, on a vu que les jachères sur les terres de sorgho repiqué surviennent le plus souvent après 
plusieurs années d'exploitations successives. La valeur assez élevée de l'élasticité des zones de cultures maraîchères et vergers s'explique quant à elle par la difficulté des investissements nécessaires pour cette culture. En effet la création d'un jardin de maraichage ou la plantation des arbres fruitiers nécessitent d'importants investissements que les paysans n'arrivent pas souvent à mobiliser [14]. De plus, la probabilité de changement d'un verger en particulier est très faible car c'est un investissement le plus souvent à long terme. Dans le même sens, les défrichements d'une brousse exigent des efforts et des moyens qui justifient les difficultés de conversion des savanes boisées.

Tableau 2. Elasticité des différents types d'utilisation de l'espace résultant de la calibration

\begin{tabular}{|c|c|c|}
\hline Utilisation de l'espace & Elasticité & Commentaire explicatif \\
\hline Habitation & 1 & $\begin{array}{l}\text { Changement autorisé dans une seule } \\
\text { direction }\end{array}$ \\
\hline Maraichage/verger & 0.8 & $\begin{array}{l}\text { Changement difficile, car investissement à } \\
\text { long terme }\end{array}$ \\
\hline Culture pluviale & 0.2 & $\begin{array}{l}\text { Pas de contrainte spécifique pour le } \\
\text { changement }\end{array}$ \\
\hline Sols nus & 0.2 & $\begin{array}{l}\text { Pas de contrainte spécifique pour le } \\
\text { changement }\end{array}$ \\
\hline Sorgho repiqué & 0.4 & $\begin{array}{l}\text { Changement un peu difficile du fait des sols } \\
\text { dédiés à la culture }\end{array}$ \\
\hline Brousse & 0.7 & $\begin{array}{l}\text { Contrainte due aux efforts et moyens à } \\
\text { fournir pour défricher }\end{array}$ \\
\hline Montagne & 1 & $\begin{array}{l}\text { Cette utilisation de l'espace est statique au } \\
\text { cours de la simulation }\end{array}$ \\
\hline
\end{tabular}

\section{3. Validation}

La validation est une mesure des performances du modèle. Elle consiste à comparer les résultats de prédiction du modèle avec la réalité ou avec les résultats d'un autre modèle. La validation constitue généralement le point faible de la plupart des modèles de changement d'utilisation de l'espace. Pourtant c'est une étape importante du processus de modélisation dans la mesure où elle permet de mieux comprendre la réalité des processus et les caractéristiques ou comportement du modèle développé ([3]; [18]).

La figure 4 illustre comment la modélisation permet de passer successivement du paysage produit par un système de gestion vers les différents modèles (spatiaux, prédictifs et dynamiques). Elle montre également que la validation de notre modèle se 
fait en trois principales étapes : la validation des résultats de l'analyse d'image [5], la validation des modèles de prédiction de type logistique [6] et la validation du modèle de simulation qui est décrite dans ce paragraphe. Les performances du modèle ont été mesurées avec deux techniques et les résultats comparés avec d'autres modèles similaires appliqués dans différentes régions dans le monde comprenant l'Europe, les Etats-Unis et l'Asie. Les deux paragraphes suivants présentent une synthèse des résultats de cette comparaison qui a été publié dans [19].

\section{Comparaison au modèle NULL à plusieurs résolutions}

Le modèle NULL est un modèle imaginaire qui prédit la persistance c'est-à-dire qui suppose qu'aucun changement n'est observé entre 1987 et 1999. Le principe des méthodes de validation à plusieurs résolutions repose sur l'idée selon laquelle une seule résolution n'est pas suffisante pour décrire des structures complexes. Le résultat de la prédiction du modèle est comparé au résultat de l'analyse d'image de la même année (1999). La procédure proposée par Costanza [3] permet de quantifier la similarité ou coefficient d'ajustement $\left(F_{w}\right)$ des structures entre deux scènes. Soit une fenêtre de taille $\mathrm{w}$ centrée sur une cellule, $\mathrm{F}_{\mathrm{w}}$ est défini par la formule suivante:

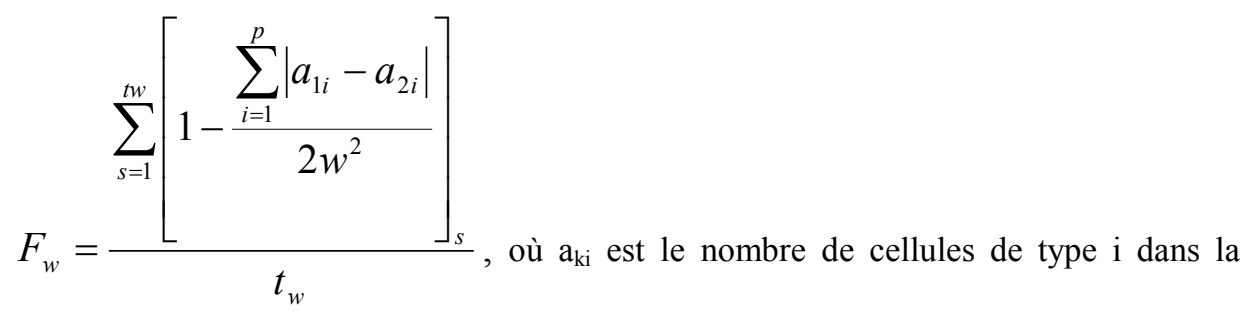

scène $\mathrm{k}$ pour la fenêtre de calcul, $\mathrm{p}$ est le nombre de type différents et $\mathrm{t}_{\mathrm{w}}$ est le nombre total de fenêtre de la scène pour une taille w donnée. Le coefficient d'ajustement global entre les deux scènes noté $F_{t}$ est une moyenne pondérée des coefficients d'ajustement pour différentes tailles de fenêtre où un poids plus important est accordé aux fenêtres de petite taille sans toutefois ignorer les fenêtres plus grandes. $F_{t}$ est calculé en utilisant la formule suivante :

$F_{t}=\frac{\sum_{w=1}^{n} F_{w} e^{-k(w-1)}}{\sum_{w=1}^{n} e^{-k(w-1)}}$ où k est une constante indiquant le poids donné aux fenêtres de

faible taille par opposition aux fenêtres de grande taille. La figure 6a montre que la courbe des coefficients d'ajustement pour le modèle de prédiction augmente d'abord 
très rapidement avant de se stabiliser autour de la valeur 1, indiquant que les structures correspondent mieux, même si le coefficient d'ajustement initial pour la fenêtre de taille 1 est relativement faible $(0,67)$. Par contre, la courbe pour le modèle NULL qui prédit la persistance commence à la même valeur mais se stabilise très vite autour de la valeur 0,82 . Un coefficient de similarité global $\left(F_{t}\right)$ peut être calculé en utilisant les différentes valeurs de $\mathrm{F}_{\mathrm{w}}$. Le coefficient de similarité global pour le modèle de prédiction est évalué à $F_{t}=0,749$ contre $F_{t}=0,725$ pour le modèle NULL. La technique de validation décrite dans [18], consiste à comparer les proportions de pixels correctement prédits par le modèle à plusieurs résolutions. La figure $6 \mathrm{~b}$ montre que l'on obtient des résultats similaires à ceux de la procédure de [3].

L'exercice de comparaison avec différents modèles a montré qu'à fine résolution, il peut arriver que le modèle NULL soit plus précis que le modèle de prédiction. Ceci dépend le plus souvent des quantités de changements observées. Ainsi, 6 des 13 modèles comparés qui affichent moins de $10 \%$ des changements observés ont plus d'erreurs que le modèle NULL [19]. Le modèle développé pour la région de Maroua est parmi les 7 applications qui sont plus précises que le modèle NULL à fine résolution.

\section{Comparaison simultanée des cartes de référence et de prédiction}

La comparaison simultanée des trois cartes disponibles sous un SIG fournit des informations complémentaires sur le comportement du modèle. La carte obtenue permet ainsi d'apprécier visuellement la source des erreurs de prédiction. On distingue ainsi les cinq catégories de pixels suivantes représentant les erreurs ou les bonnes prédictions :

1. les zones où le changement est observé et le modèle prédit la persistance ;

2. les zones où le changement est observé et le modèle prédit le changement c'est à dire que le modèle prédit correctement le changement;

3. les zones où le changement est observé et le modèle prédit le changement mais vers la mauvaise catégorie ;

4. les zones où la persistance est observée et le modèle prédit le changement ;

5. les zones où la persistance est observée et le modèle prédit la persistance c'est à dire les zones où le modèle prédit correctement la persistance.

Soient A, B, C et D les proportions des catégories respectives 1, 2, 3 et 4. Le niveau de mérite est une statistique qui représente le ratio entre la proportion correctement prédite et la proportion totale des changements observés et prédits $(B /(A+B+C+D))$. $C e$ niveau a été évalué à $24 \%$ pour le modèle de la région de Maroua. La figure 7 montre dans l'ensemble que les applications où on a une proportion importante de changements observés ont tendance à afficher une précision de prédiction plus grande telle que mesurée par le niveau de mérite. Toutes les 6 applications qui ont un niveau de mérite 
inférieur à $15 \%$ affichent une proportion de changement observé inférieure à $10 \%$. Seule l'application du Périnet sur les 13 applications considérées affiche un niveau de mérite supérieur à $50 \%$.
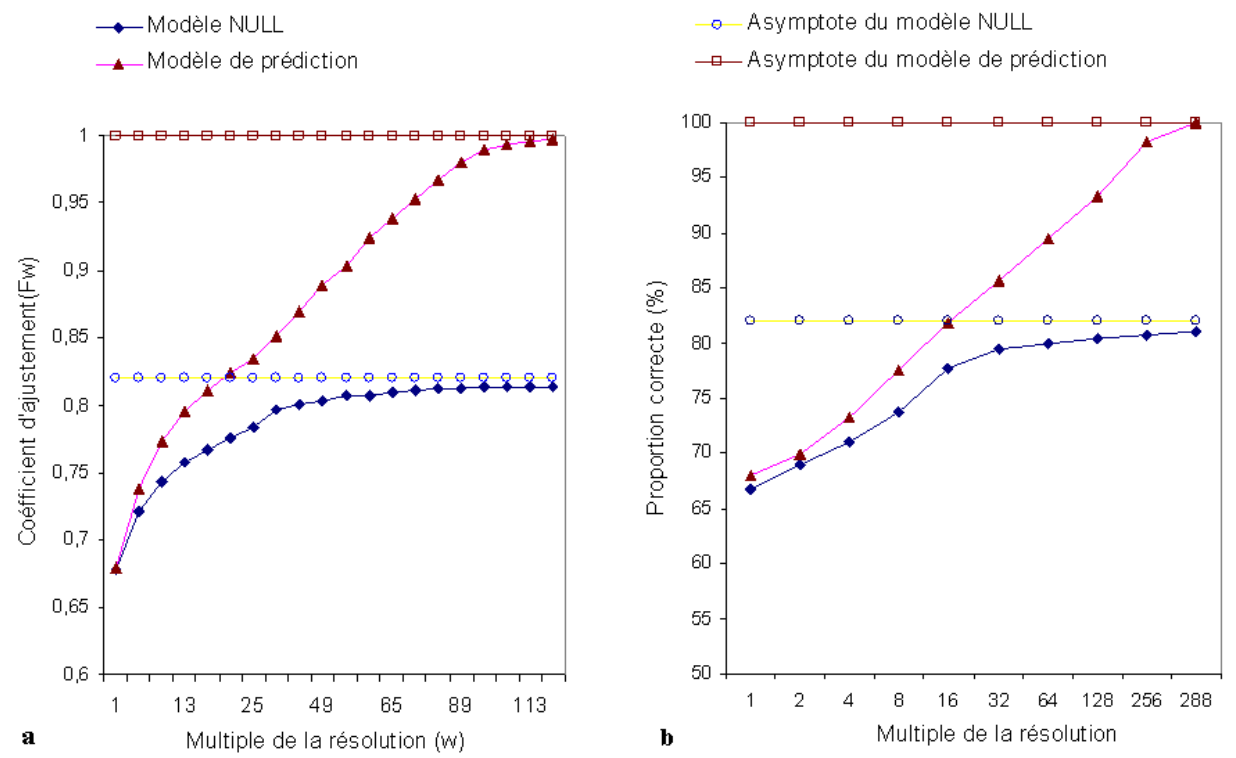

Figure 6. Comparaison des performances du modèle de prédiction avec celles du modèle NULL à plusieurs résolutions. a) coefficients de similarité des structures entre la carte observée et prédite b) proportion de pixels correctement prédits.

La précision du producteur est la proportion de pixels que le modèle prédit correctement comme changement sachant que les cartes de référence indiquent un changement $(\mathrm{B} /(\mathrm{A}+\mathrm{B}+\mathrm{C}))$. La précision d'utilisateur est la proportion de pixels que le modèle prédit correctement comme changement sachant que le modèle prédit le changement $(B /(B+C+D))$. Dans [19], nous analysons les précisions de producteur et d'utilisateur pour les 13 applications. La précision du producteur pour le modèle de la région de Maroua est évaluée à $40 \%$ et la précision d'utilisateur est évaluée à $32 \%$. Ces résultats signifient en d'autres termes que le modèle prédit correctement $40 \%$ des changements qui ont effectivement eu lieu. De la même façon il prédit correctement $32 \%$ des pixels qu'il prédit comme changement. La précision du producteur est supérieure à $50 \%$ pour 3 applications (Perinet, Honduras, et Costa Rica). La précision d'utilisateur est également supérieure à $50 \%$ pour 3 applications (Perinet, Haidian, et Costa Rica).

ARIMA JournaI 
Les résultats de validation du modèle permettent entre autre d'identifier les zones où la cartographie ne permet pas de mieux capturer la réalité.

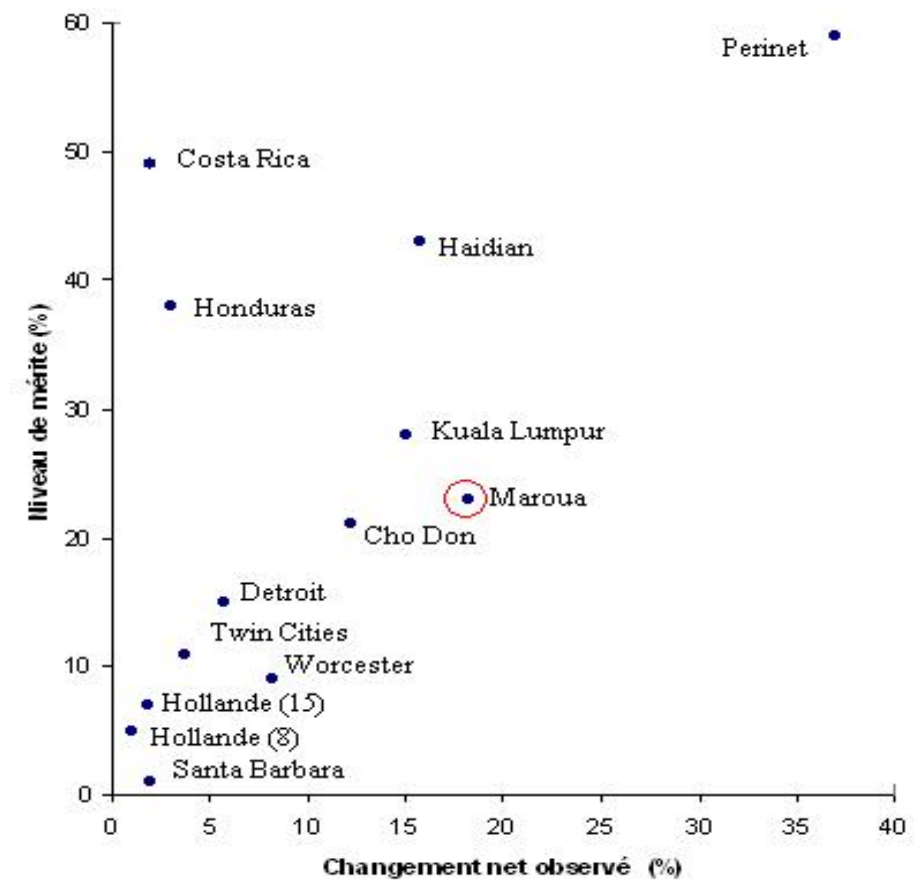

Figure 7. Relation entre le niveau de mérite et la proportion de changements observés [19].

\section{Résultats de la simulation des scénarios formulés}

Une analyse des dynamiques du système agraire effectuée sur la base des hypothèses formulées à la section 3 de cet article [8], nous a conduit à trois principaux scénarios résumés dans le tableau 3 et dont les résultats de simulation sont présentés dans cette section.

\subsection{Scénario 1 considérant le maintien des tendances observées dans le passé}

Le scénario de base considère que la stratégie d'utilisation de l'espace actuelle basée sur un système extensif se poursuivra au cours des prochaines années. On suppose que la 
demande d'allocation des superficies des zones d'habitation, des zones de cultures maraîchères, de sorgho repiqué, et de savanes boisées évoluent suivant une fonction linéaire avec le même rythme que celui observé dans le passé (Figure $8 \mathrm{a} ; 7 \mathrm{~d}$ et 7e).

Pour chaque type d'utilisation de l'espace LUi, la demande pour une année $\mathrm{j}$ est théoriquement donnée par la fonction géométrique suivante :

$$
L U_{i j}=L U_{i 0}\left[1+d^{*}\left(\frac{\Delta L U i}{n L U_{i 0}}\right) * 100\right]^{j} \text { où } L U_{i 0} \text { représente la quantité du type }
$$

d'utilisation i au début de la simulation, $\Delta L U i$ est le changement total sur toute la période de simulation représenté par la valeur $\mathrm{n}$ et $\mathrm{d}$ détermine la direction du changement avec $d=+1$ pour un accroissement et $d=-1$ pour une réduction. Les valeurs de $L U_{i j}$ sont calculées automatiquement de telle façon que la superficie totale des différentes utilisations de l'espace soit égale à la superficie de la zone d'étude.

Les hypothèses pour l'évolution des superficies de cultures pluviales et les sols nus sont les mêmes dans les trois scénarios. Les superficies des cultures pluviales augmentent pour quelques années avant de se stabiliser à un maximum qui correspond au fait que les défrichements pour l'extension agricole ne sont plus possibles. Ces superficies baissent ensuite parce qu'elles sont converties en fonction des potentialités des terres et des stratégies paysannes pour l'extension de la culture du sorgho repiqué et dans une moindre mesure le développement des cultures maraîchères. Cette situation rejoint les résultats obtenus par Njomaha (2004) qui a identifié parmi les principaux changements agraires dans la région, l'abandon de plusieurs cultures pluviales à long cycle du fait de la réduction de la saison pluvieuse. La tendance observée de récupération progressive des sols nus (dégradés) pour les activités agricoles et le reboisement continue. Aucune supposition n'est faite sur le rythme de récupération des sols nus ou de conversion des zones de culture pluviale. En effet, les excédents et les déficits causés par l'extrapolation des autres utilisations de l'espace sont alloués à ces deux classes proportionnellement à leur taille au début de la simulation.

Les simulations dont le résultat est illustré à la figure $9 \mathrm{~b}$ montrent que les zones d'habitation et de maraichage/verger augmentent mais les superficies restent relativement faibles. De très faibles extensions de la culture maraîchère se font en général sur les terres alluviales qui peuvent également être utilisées pour les cultures pluviales. Les extensions récentes de la culture du sorgho de contre saison se font principalement au détriment des savanes boisées notamment au sud est de la zone d'étude, entre les villages Laf et Mindif. Ces zones d'extension correspondent à des vertisols qui sont très aptes à la culture du sorgho repiqué. A la fin de la simulation, les meilleures terres (classées de très bonne à assez bonne aptitude) sont presque toutes occupées par le sorgho repiqué et on commence à observer des extensions sur les terres 
alluviales. Contrairement à quelques extensions de la culture observées dans la zone des piémonts notamment aux environs de Méri dans le nord ouest de la zone d'étude, la simulation montre que les extensions sont difficiles sur les sites en micro-topographie (faibles pentes) comme à Mowo.

Tableau 3 : Synthèse des hypothèses effectuées pour chaque scenario et traduction en paramètres dans le modèle. $d$ est la direction du changement et * correspond à stable

\begin{tabular}{lrrrrrrrrr} 
& & \multicolumn{2}{c}{ Scénario 1 } & \multicolumn{2}{c}{ Scénario 2 } & \multicolumn{2}{c}{ Scénario 3 } \\
\cline { 5 - 10 } Utilisation & $\mathbf{1 9 9 9}$ & $\mathbf{d}$ & Taux & $\mathbf{2 0 1 0}$ & Taux & $\mathbf{2 0 1 0}$ & Taux & $\mathbf{2 0 1 0}$ \\
Habitation & 6675 & +1 & 0,48 & 7035 & 0,48 & 7035 & 0,48 & 7035 \\
Maraichage/verger & 4963 & +1 & 1,30 & 5719 & 0,70 & 5358 & 2,50 & 6511 \\
Culture pluviale & 128063 & -1 & 3,09 & 110953 & 3,10 & 96158 & 3,10 & 112674 \\
Sol nu & 31294 & -1 & 3,31 & 21608 & 3,30 & 22960 & 3,30 & 26996 \\
Sorgho repiqué & 93575 & +1 & 3,81 & 141159 & 5,00 & 160045 & 2,50 & 122779 \\
Brousse & 74600 & -1 & 3,11 & 52695 & 4,00 & 47613 & 2,50 & 63174 \\
Montagne & 17981 & $*$ & 0,00 & 17981 & 0,00 & 17981 & 0,00 & 17981 \\
Total & $\mathbf{3 5 7 1 5 0}$ & & & $\mathbf{3 5 7} \mathbf{1 5 0}$ & & $\mathbf{3 5 7 1 5 0}$ & & $\mathbf{3 5 7 1 5 0}$ \\
\hline
\end{tabular}

\subsection{Scénario 2 considérant l'évolution suivant une trajectoire Malthusienne}

Le scénario 2 considère que la région suivra une trajectoire qui semble prévaloir dans plusieurs sites de la zone d'étude. Il s'agit de la trajectoire de Malthus caractérisée par la dégradation des ressources naturelles et des institutions qui conduit à la famine et aux migrations [13]. Dans ce cas, la baisse de la fertilité des sols induit un accroissement plus important des superficies cultivées pour la même production. De même, avec l'absence d'institutions fortes pouvant garantir la protection des ressources naturelles, les défrichements des savanes boisées sont plus importants. Dans ce deuxième scénario, on suppose que :

a) la demande en céréales (sorgho repiqué dans ce cas) croit au même rythme que celui de la population soit environ 3,3\% par an. La dégradation des sols et l'extension de la culture observée sur des terres marginales conduisant à la baisse des rendements, 
exigent des superficies plus importantes pour répondre au même besoin. Les superficies en sorgho repiqué croissent donc à un taux de 5\% par an (Figure 8d);

b) les besoins supplémentaires des villes en fruits et légumes ne sont pas tous satisfaits à cause des difficultés d'investissement. Les superficies utilisées pour le maraichage/verger augmentent comme dans le scénario 1 mais à un rythme beaucoup plus faible soit $0,70 \%$ (Figure 8a);

c) l'augmentation de la pression pour l'extension du sorgho repiqué conduit à une réduction des savanes boisées. Les populations pauvres recourent à des défrichements illégaux pour la collecte du bois de feu qui est vendu. Le rythme des défrichements observés devient plus important que dans le scénario 1 mais reste limité compte tenu du fait que le processus est en cours d'achèvement (4\%, Figure $8 \mathrm{e}$ ).

Dans le scénario 2 où la demande en superficie pour la culture du sorgho de contre saison est plus importante que la disponibilité des meilleures terres, les simulations dont le résultat est illustré à la figure $9 \mathrm{c}$ montrent que la demande en espace supplémentaire est satisfaite de plusieurs façons: d'une part, on observe des défrichements plus importants de la savane au sud de Maroua. D'autre part, on note une compétition plus importante sur les espaces où les sols de type «intermédiaire » se prêtent également aux cultures pluviales. C'est le cas également sur les vertisols qui se trouvent le long des cours d'eau. Dans la plaine du Diamaré, notamment autour du terroir de Balaza situé à environ $20 \mathrm{~km}$ à l'est de Maroua, des extensions de faibles superficies se font sur les zones de sols nus dont les terres sont pour la plupart dégradées (hardé). Ce sont des terres qui ont été par le passé utilisées pour la culture du sorgho de contre saison mais qui sont parvenues à un stade de dégradation avancé. Leur mise en valeur nécessite des aménagements tels que le binage et la mise en place ou l'entretien des diguettes. On observe aussi quelques extensions dans la zone des piémonts notamment autour de Méri et au sud du terroir de Mowo. Toutefois, cette dynamique reste limitée du fait des contraintes du milieu et des investissements qu'il faut pour les aménagements. Comme le montre la figure $8 \mathrm{a}$, le rythme d'accroissement des superficies utilisées pour le maraichage/verger est un peu plus faible que dans le scénario 1. Les simulations montrent que les nouveaux maraichage/verger se mettent en place dans la zone de Salak et autour de la localité de Ndoukoula, située au sud ouest de la zone d'étude.

On peut voir que malgré la demande importante en bois de feu et en espace pour l'extension de la culture du sorgho de contre saison, certaines zones très proches des centres urbains restent relativement bien boisées. Ce sont notamment la zone de Balaza et la zone autour de Mindif. Les observations de terrain couplées avec l'exploitation de la carte des sols ont montré que les terres de ces zones ne sont pas aptes à l'agriculture. De plus, les espèces ligneuses présentes dans ces zones ne seraient pas pour la plupart très appréciées comme bois pour le feu. Elles ne contribuent pas donc significativement à l'approvisionnement actuel de la ville de Maroua en bois de feu, mais constituent des 
réserves importantes de bois pour les populations rurales environnantes et des zones de pâturage pour le bétail.
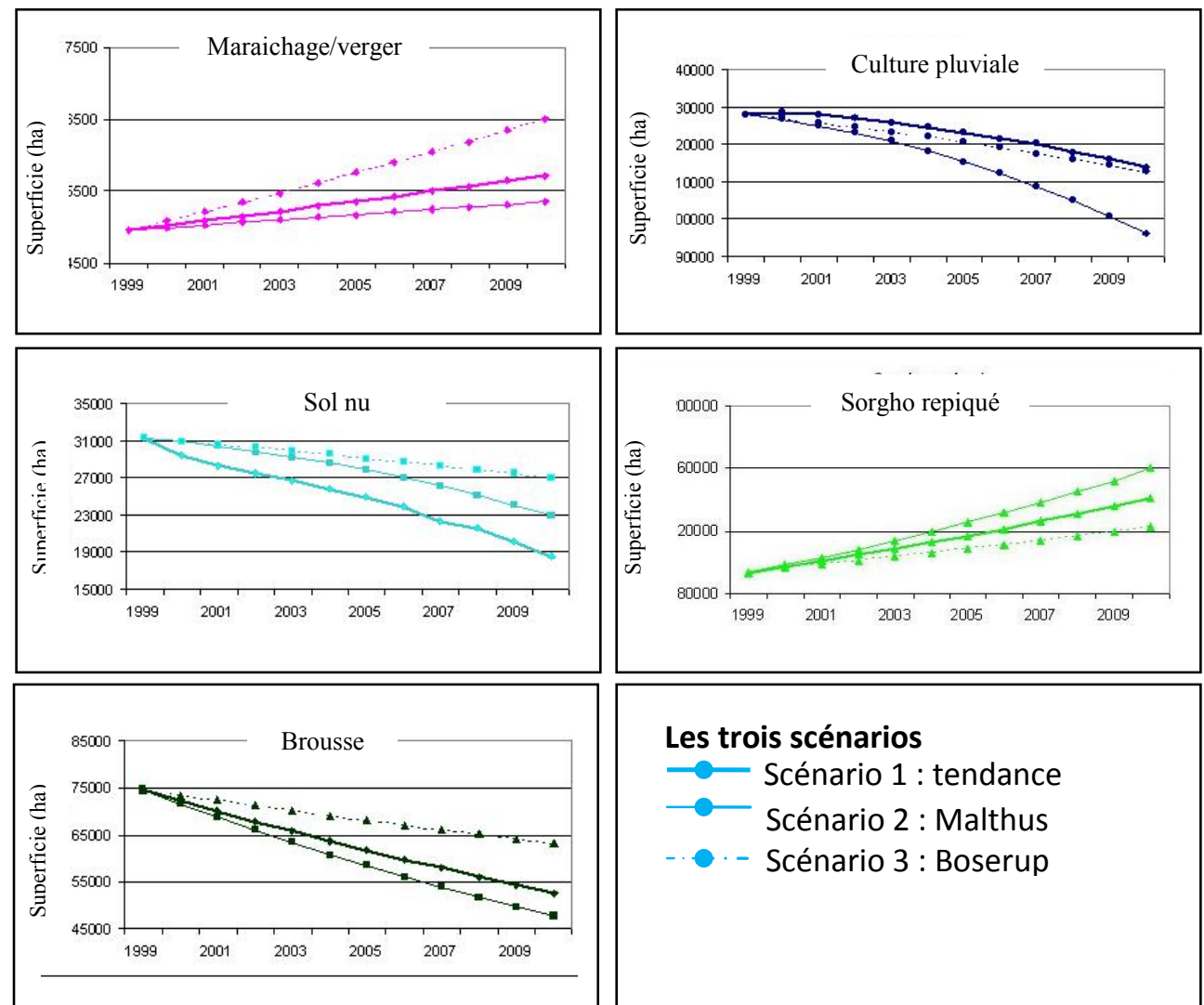

\section{Les trois scénarios}

- Scénario 1 : tendance

- Scénario 2 : Malthus

- Scénario 3 : Boserup

Figure 8. Evolution des superficies des différents types d'utilisation de l'espace pour les trois scénarios. 


\subsection{Scénario 3 considérant l'évolution suivant une trajectoire Boserupienne}

Le scénario 3 considère qu'une véritable transition au sens de Boserup se mettra en place. En effet, la croissance de la population va entrainer des innovations et les acteurs vont investir de plus en plus pour améliorer la fertilité des sols. Dans cette même perspective optimiste, des mesures de conservation seront prises pour limiter la destruction massive des savanes boisées pour l'extension de la culture du sorgho repiqué, notamment par le renforcement des mesures de protection des réserves forestières. Ainsi, les populations auront besoin de moins d'espace pour la même production. On suppose dans ce scénario que :

a) les superficies utilisées pour le maraichage/verger augmentent au rythme de 2,5\% parce que des investissements plus importants sont accordés au développement de la culture maraîchère et la plantation des arbres fruitiers (Figure $8 \mathrm{a}$ );

b) les superficies de sorgho repiqué continuent d'abord à augmenter avec un taux d'accroissement beaucoup plus faible (2,5\%) que dans le scénario 1 et 2 (Figure $8 \mathrm{~d}$ ). Ceci s'explique par le fait qu'on assiste à une adoption progressive des techniques d'intensification de la culture de sorgho repiqué. Si les mesures d'intensification sont maintenues, ces superficies devraient se stabiliser et tendre à la baisse dans les phases ultérieures de l'évolution du système ;

c) le rythme des défrichements baisse également à 2,5\% par an sous l'influence des politiques forestières qui sont mises en place pour la protection et l'aménagement des ressources ligneuses (Figure 8e). Les mesures prises et les moyens consentis par l'administration forestière à cet effet devraient amener les superficies à se stabiliser et augmenter si les investissements sont soutenus dans le temps.

Dans le scénario 3, les simulations dont le résultat est illustré à la figure $9 \mathrm{~d}$ montrent que les cultures maraîchères et la plantation des arbres fruitiers prennent de l'importance et les augmentations de superficies sont observées autour du principal centre urbain et des principales agglomérations environnantes. Toutefois, les superficies occupées restent faibles à l'échelle régionale et sont localisées à proximité des cours d'eau. Malgré les besoins supplémentaires, on constate que la zone entre Laf et Mindif au sud de Maroua n'est pas sollicitée pour l'extension du maraichage/verger. Ceci se confirme par la réalité sur le terrain et s'explique par le fait que les ressources en eau souterraine dans cette localité sont réduites.

On constate par contre que les zones de savanes boisées restantes dans cette zone sont relativement bien conservées. Aucune restriction représentant une quelconque politique de conservation n'a été incluse dans le modèle. Ce scénario pourrait donc être affiné en interdisant les défrichements dans les réserves forestières et des zones de 
brousse bien identifiées. Celles-ci peuvent être des espaces que les populations s'engagent à exploiter comme forêt communautaire pour l'approvisionnement local et la vente du bois de feu.
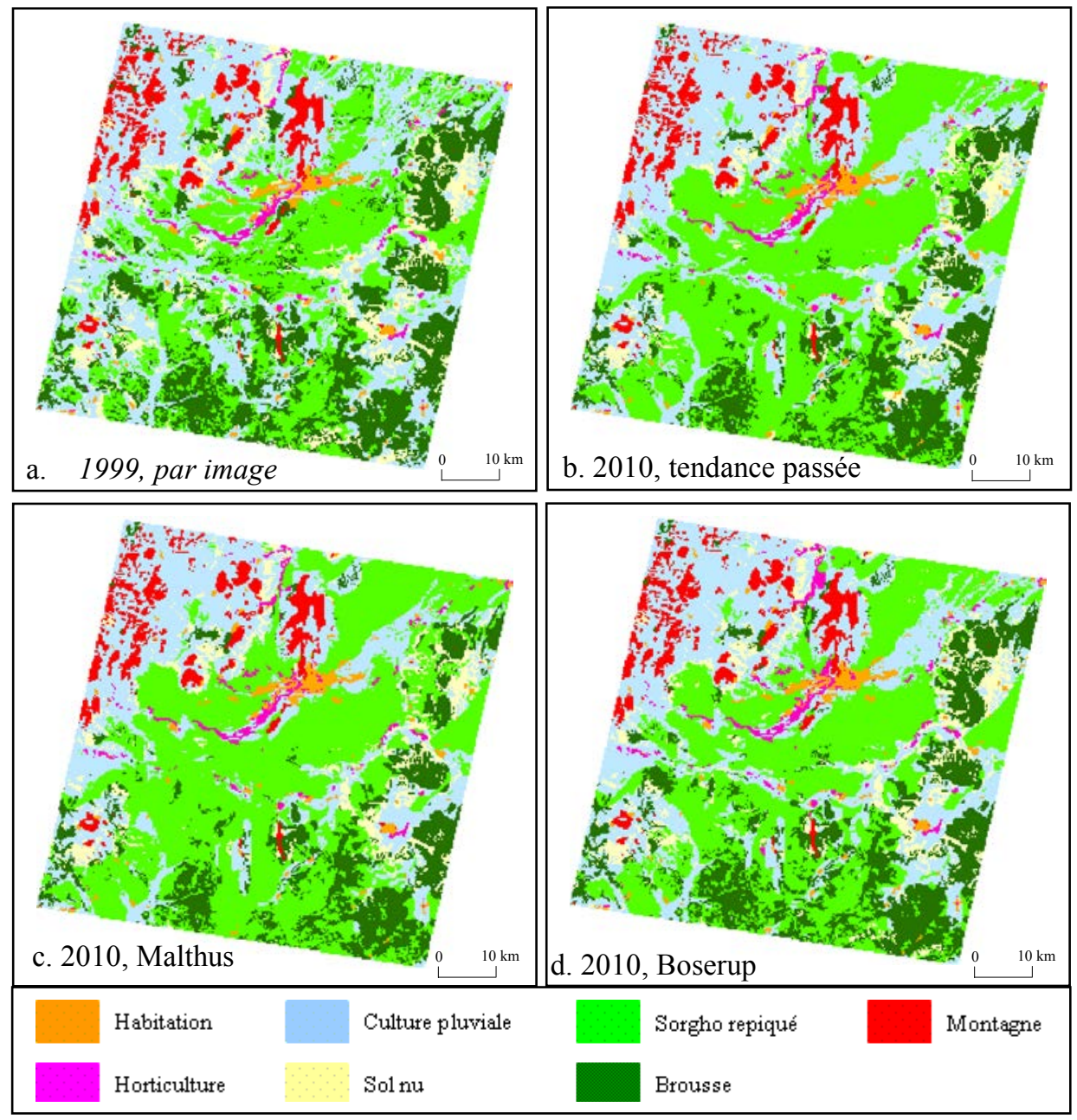

Figure 9. Carte d'utilisation de l'espace a) obtenue par analyse d'image et carte prédite pour les scénarios b) de maintien de la tendance passée et des trajectoires c) de Malthus et d) de Boserup 


\subsection{Cas d'utilisation du modèle}

Le modèle développé est un outil approprié pour un observatoire des dynamiques agraires et du développement rural. Il peut être utilisé par une diversité d'acteurs (chercheurs, gestionnaires de l'espace, décideurs et populations locales) impliqués dans la planification et l'aménagement de l'espace pour guider la prise de décision et opérer une gestion adaptative. Les trois scénarios explorés dans cet article ont été formulés sur la base des résultats de recherche pour tester des hypothèses sur les trajectoires du système d'utilisation de l'espace [14]. L'application du modèle à l'ensemble de la région permettrait une comparaison plus fine avec les résultats d'analyse socio-économiques et historiques à l'échelle des villages. Avec les institutions chargées de la gestion et de la planification de l'utilisation de l'espace (aménagement du territoire, agriculture, élevage, forêts et environnement), des scénarios adaptés au contexte de la prise de décision peuvent être formulés. Deux exemples concrets d'application concernent le processus de création d'une forêt communautaire et l'adoption des mesures de conservation des aires protégées qui sont en cours de dégradation. Les choix pourront être inclus dans la simulation comme des règles de gestion et les impacts de chaque mesure pourront être appréciés dans l'espace. Les résultats des simulations pourront susciter la discussion autour de l'allocation des terres et assister ainsi dans les choix de zonage de l'utilisation l'espace. Une utilisation peut également être envisagée avec les acteurs locaux pour évaluer l'impact de certaines mesures ou choix de gestion de l'espace. Il s'agit de susciter les discussions et préparer la concertation ou la négociation dans le cadre de démarches participatives d'utilisation et de gestion de l'espace. Le modèle devenant dans ces cas, un objet intermédiaire de cognition collective

\section{Conclusion et perspectives}

Le modèle développé a permis d'explorer les processus de changement d'utilisation de l'espace sur la petite région autour de Maroua. Les conditions et les impacts de l'évolution du système agraire ont été explicités suivant différents scénarios déterminés par les réponses des populations aux mutations observées dans la zone d'étude. Les deux principaux processus mis en exergue sont : 1) le développement d'un système extensif caractérisé par les défrichements importants pour l'extension agricole, 2) la transition vers un système plus intensif caractérisé par l'émergence des cultures maraîchères ou des arbres fruitiers et la mise en place de mesure de conservation des ressources (sols; ligneux, etc.). Pour chaque scénario, les résultats des simulations montrent les zones les plus affectées par les défrichements, les zones où on peut

ARIMA Journal 
s'attendre à l'extension du sorgho repiqué, des cultures maraîchères ou la plantation d'arbres fruitiers. Les cartes produites montrent également les zones boisées qui pourraient encore faire l'objet d'actions de conservation, de gestion ou d'exploitation plus durables. Le modèle ainsi construit constitue un support d'aide à la planification de l'utilisation de l'espace.

Les résultats de l'analyse des scénarios peuvent être utilisés par les gestionnaires de l'espace et les autres acteurs pour modifier la structure et les fonctions du paysage. Toutefois, les résultats obtenus à chaque étape de la construction et de l'exécution du modèle ont mis en exergue certaines limites qui ouvrent des perspectives d'amélioration ou d'adaptation. Ces limites peuvent être classées sous les trois thèmes suivants : le changement d'échelle, la collecte et la représentation des données, l'approche de modélisation.

Une première façon d'améliorer le modèle consisterait à explorer les effets du changement de résolution spatiale des données sur la précision des prédictions. Une deuxième possibilité consisterait à prendre en compte les spécificités des différentes zones agro écologiques en définissant des modèles de régression propres à chaque zone. D'autre part, l'approche de modélisation utilisée fait que les résultats obtenus ne sont valables que pour la région autour de Maroua. Toutefois la méthodologie suivie est en tout point applicable à des espaces plus vastes comme l'ensemble de la zone des savanes d'Afrique Centrale. La principale contrainte reste le problème de manque de données spatiales explicites sur tous les facteurs qui sont importants dans l'analyse des processus de changement d'utilisation de l'espace.

Les résultats de la validation ont montré que les performances du modèle dépendent de la qualité du processus de préparation des données et de leur format de représentation. En particulier, le choix des résolutions spatiale et temporelle déterminent fortement la pertinence et la précision du modèle. Pour les données d'utilisation de l'espace obtenues par télédétection, la méthode de cartographie doit minimiser les erreurs de confusion des classes d'occupation du sol. De façon générale, les données spatiales doivent représenter au mieux les processus étudiés. L'indisponibilité où la difficulté de disposer des données spatiales explicites pour certains facteurs de type socio-économiques ou liés aux motivations, logiques ou stratégies des acteurs constitue une limite du modèle développé. Un effort important est nécessaire pour développer des bases de données spatiales explicites intégrant les données socio-démographiques et culturelles plus pertinentes.

La difficulté de prendre en compte les aspects socio-démographiques et culturelles dans le modèle n'est pas seulement liée à la disponibilité des données mais constituent une limite inhérente à l'approche de modélisation. La manière dont les relations de causalité et les mécanismes de changement sont pris en compte dans le modèle fait que certains aspects qui sont pourtant déterminants ne sont pas pris en compte dans le modèle. Il peut s'agir de facteurs dont les dynamiques ne peuvent être facilement 
représentées sous forme spatiale ou les effets de leur interaction ne sont pas toujours visibles dans l'espace. Une solution consiste à s'orienter vers des modèles utilisant des approches déductives qui présentent l'avantage de conduire aux meilleures chaînes de causalité et d'explication que les approches inductives.

Les approches de types multi-agents couplées aux Systèmes d'Information Géographique semblent appropriées pour répondre à certaines de ces préoccupations liées à la complexité du système agraire. La modélisation multi-agents se présente comme une approche prometteuse et complémentaire. Elle offre des possibilités qui permettraient de répondre à un nombre important de questions concernant notamment la modélisation des processus de prise de décision individuelle, l'influence des facteurs politiques et institutionnels tels que le système foncier, les dynamiques de marché et les pratiques traditionnelles d'utilisation et de gestion des terres.

\section{Bibliographie}

[1] BAKER W.L., 1989. A review of models of landscape change. Landscape ecology 2:11-133.

[2] BOSERUP E., 1965. The conditions of Agricultural Growth : the economic of agrarian change under population pressure. (Ed) George Allenand Unwin LTD London, Aldine Publishing company, Chicago, 122 pages.

[3] COSTANZA R., 1989. Model Goodness of fit: a multiple resolution procedure. In Ecological modelling 47 (1989) 199-215.

[4] FOTSING E. and VERBURG P.H., 2001. A spatially explicit and empirical approach for analysing land use change in the Far North region of Cameroon. In Madi A., Loth P., Bauer H. And De Iongh H. (eds), 2001. Proceedings of the International Conference on the Management of fragile Ecosystem, 13-16 November 2000, Maroua-Cameroon, pp 105-119.

[5] FOTSING E., CHEYLAN J-P., and VERBURG P.H, 2006. From images to patterns: a step toward the identification and modelling of land change processes in a savannah landscape. Proceedings of the $6^{\text {th }}$ AARSE International Conference on Earth Observation \& Geoinformation Sciences for Africa's Development, Cairo-Egypt, 30 October - 2 November 2006.

[6] FOTSING E., VERBURG P.H, DEGROOT W.T., TCHUENTE M and CHEYLAN J-P., 2007. Une méthode d'exploration spatiale pour analyser les facteurs déterminant les changements d'utilisation de l'espace appliquée à une région du Nord Cameroun. Proceedings of the International Conference on the theme "Central Africa, Cameroon and Global Change ", organised by IGBP Cameroon, Yaounde, 6 - 8 Juin 2007.

[7] FOTSING E., VERBURG P.H, DEGROOT W.T., CHEYLAN J-P., TCHUENTE M., 2010. Un modèle intégré pour explorer les trajectoires d'utilisation de l'espace en zone de savanes sèches du Cameroun. Actes du colloque "Savanes africaines en développement : innover 
pour durer », Garoua, Cameroun, 20 - 23 Avril 2009. Prasac, N'Djaména, Tchad : CIRAD, Montpellier, France, Cédérom.

[8] FOTSING E., 2009. Un Système d'Information pour l'analyse intégrée des changements d'utilisation de l'espace en zone des savanes de l'Extrême Nord du Cameroun. Thèse de PhD, Université de Leiden Pays-Bas, à paraître.

[9] LAMBIN E. F., 1994. Modelling deforestation processes : A review. Research report $\mathrm{n}^{\circ} 1$, TREES Series. 113 pages.

[10] LAMBIN F. E., GEIST H. J., and Lepers E., 2003. Dynamics of Land use and Land cover change in tropical regions. Annu. Rev. Environ. Ressour. 2003. 28:205-41.

[11] MADI, A. HUUB P. et SALI B., 2003. La demande urbaine en bois énergie et nécessité d'une gestion rationnelle des ressources naturelles : le cas de la ville de Maroua à l'Extrême Nord du Cameroun. In Jamin J.Y., Seiny Boukar L. et Floret C. (Eds), 2003. Savanes africaines : des espaces en mutation, des acteurs face à de nouveaux défis. Actes du colloque, mai 2002, Garoua, Cameroun. PRASAC, 9 pages.

[12] NIEMEIJER D., and MAZZUCATO V., 2002. Population growth and the environment in Africa: local informal institutions, the missing link. Amsterdam Research Institute for Global Issues and Development Studies. pp. 171-193.

[13] MILLEVILLE P., et SERPANTIE G., 1994. Intensification et durabilité des systèmes agricoles en Afrique soudano-sahélienne. In Actes du séminaire sur la promotion de systèmes agricoles durables dans les pays de l'Afrique soudano-sahélienne, FAO/CIRAD, 10-14 janvier 1994, Dakar, Sénégal, pp. 33-45.

[14] NJOMAHA C., 2004. Agricultural change, food production and sustainability in the far North of Cameroon. Phd Thesis, Leiden University, 245 pages.

[15] PARKER D.C., MANSON S.M., JANSSEN M.A., HOFFMAN M. and DEADMAN P., 2003. Multiagent systems for the simulation of Land-Use and Land-Cover Change : A review. Annals of the Association of American Geographers 93 (2) : 314-337.

[16] PELISSIER P., 1995. Transition foncière en Afrique noire : Du temps des terroirs au temps des finages. In Dynamique des systèmes agraires. Terre, Terroir, territoire: Les tensions foncières. Edition ORSTOM, collection colloques et séminaires, pp. 19-34.

[17] PIERI C., 1989. Fertilité des terres de savanes: bilan de trente ans de recherche et de développement agricole au sud du Sahara. CIRAD, 444 pages.

[18] PONTIUS R. G. J., HUFFAKER D. and DENMAN K., 2004. Useful techniques of validation for spatially explicit land-change models. Ecological Modelling 179(4): 445-461.

[19] PONTIUS. R. G. J., WIDEKE B., CATELLA J-C., KEITH C., TON DE NIJS, DIETZEL C., ZENGQIANG D., FOTSING E., GOLDSTEIN N., KOK K., KOOMEN K., LIPPITT C. D., McCONNEL W., PIJANOWSKI B., SNEBAL P., SOOD A. M., SEAN SWEENEY, TRAN NGOC T., VELDKAMP A. T., and VERBURG P. H., 2007. Comparing the input, output, 
and validation maps for several models of land change. Annals of Regional Science, DOI 10.1007/s00168-007-0138-2.

[20] RGPH, 1987. Demo 87 : Deuxième recensement général de la population et de l'habitat du Cameroun. Volume II, Résultats Bruts, 838 pages.

[21] RIEBSAME W.E., PARTON W.J., GALVIN K.A., BURKE I.C., BOHREN L., YOUNG R. and KNOP E., 1994. Integrated modeling of land use and cover change: A conceptual scheme for applying an integration strategy to agricultural land use on the U.S great plains. In Biosciences vol.44. No.5, pp. 350-356.

[22] SEIGNOBOS C., IYEBI-MANDJECK O. et ABDOURAHMAN N., 1995. Saturation foncière et Muskuwari : Terroir de Balaza-Domayo. Rapport DPGT, 62 pages+Annexes.

[23] VELDKAMP A., and FRESCO L. O., 1997. Exploring Land use scenarios, an alternative approach based on actual land use. Agricuktural systems, 55. No 11. pp 1-77.

[24] VERBURG P.H., SOEPBOER W., VELDKAMP A., ESPALDON V., and Sharifa M., 2002. Modelling the spatial dynamics of regional land use : the CLUE-S model. Environmental Management vol 30 No 3 pp. 391-405. 